\title{
Robust Bilayer Charge Pumping for Spin- and Density-Resolved Quantum Gas Microscopy
}

\author{
Joannis Koepsell, ${ }^{1,2, *}$ Sarah Hirthe, ${ }^{1,2}$ Dominik Bourgund, ${ }^{1,2}$ Pimonpan Sompet ${ }^{1,2}$ Jayadev Vijayan, ${ }^{1,2}$ \\ Guillaume Salomon $\odot^{1,2}$ Christian Gross $\oplus^{1,2,3}$ and Immanuel Bloch ${ }^{1,2,4}$ \\ ${ }^{1}$ Max-Planck-Institut für Quantenoptik, 85748 Garching, Germany \\ ${ }^{2}$ Munich Center for Quantum Science and Technology (MCQST), 80799 München, Germany \\ ${ }^{3}$ Physikalisches Institut, Eberhard Karls Universität Tübingen, 72076 Tübingen, Germany \\ ${ }^{4}$ Fakultät für Physik, Ludwig-Maximilians-Universität, 80799 München, Germany
}

(Received 18 February 2020; accepted 9 June 2020; published 2 July 2020)

\begin{abstract}
Quantum gas microscopy has emerged as a powerful new way to probe quantum many-body systems at the microscopic level. However, layered or efficient spin-resolved readout methods have remained scarce as they impose strong demands on the specific atomic species and constrain the simulated lattice geometry and size. Here we present a novel high-fidelity bilayer readout, which can be used for full spin- and densityresolved quantum gas microscopy of two-dimensional systems with arbitrary geometry. Our technique makes use of an initial Stern-Gerlach splitting into adjacent layers of a highly stable vertical superlattice and subsequent charge pumping to separate the layers by $21 \mu \mathrm{m}$. This separation enables independent highresolution images of each layer. We benchmark our method by spin- and density-resolving twodimensional Fermi-Hubbard systems. Our technique furthermore enables the access to advanced entropy engineering schemes, spectroscopic methods, or the realization of tunable bilayer systems.
\end{abstract}

DOI: 10.1103/PhysRevLett.125.010403

Quantum simulation has opened a new and unique window to explore static and dynamical properties of quantum matter [1-5], which is difficult to access with classical numerical computations. One important class of strongly correlated materials can be simulated with FermiHubbard systems, in which the intricate interplay between density (charge) and spin degrees of freedom is believed to contain essential ingredients for the physics of hightemperature superconductivity [6,7]. Despite its simple form, the two-dimensional Fermi-Hubbard model still poses major challenges to the exploration of its phase diagram. In addition, it is an open question to what extent the layered structure in real materials like the cuprates affects the resulting physical properties [8,9].

Quantum gas microscopy of two-dimensional FermiHubbard systems promises to shed new light on the interplay between antiferromagnetic order and mobile density (charge) dopants. Several aspects were recently explored, such as long-range antiferromagnetic correlations [10], spin and density transport [11,12], or the effect of doping on two-point spin correlations [13]. However, most

Published by the American Physical Society under the terms of the Creative Commons Attribution 4.0 International license. Further distribution of this work must maintain attribution to the author(s) and the published article's title, journal citation, and DOI. Open access publication funded by the Max Planck Society. experiments are strongly constrained in their accessible observables. It is usually possible to measure either density observables, without being able to distinguish between doublons or holes (parity projection) [14,15], or single spin-component observables alone [16,17], thereby severely restricting the potential of quantum gas microscopy, especially for doped systems. Recent studies at full density- and spin-resolution have shown the capability of simultaneous detection of occupation and spin, including the static $[18,19]$ or dynamic [20] aspects of spin-charge separation in one dimension or magnetic polarons in two dimensions [21]. However the applied technique [22] typically strongly constrains available lattice geometries, reduces system size and leads to weaker couplings in the system.

Alternative methods for spin-resolved readout are based on vector light shifts or electronic dark states and can be partly used to overcome such deficiencies [23,24], however, they were demonstrated for atomic species not available for Fermi-Hubbard experiments. In addition, it would be desirable to extend quantum gas microscopy techniques beyond two dimensions, thus enabling the control and study of multilayered systems. This is highly challenging, because the high density of the analyzed systems, on scales of the optical resolution, prevents the employment of multilayer readout schemes that are suitable for systems with large lattice spacing and vanishing tunnel coupling between sites [25-27]. For bosons, a first example of such a scheme has been demonstrated [28]. 
Here we demonstrate a novel approach that overcomes all these challenges. It allows us to realize and image bilayer systems and to obtain full spin- and densityresolved images of two-dimensional quantum gases with arbitrary geometries, including the two-dimensional FermiHubbard systems studied here. In contrast to previous highfidelity spin-resolution techniques [22], our scheme can be readily applied to nonlattice systems, by employing an ancillary detection lattice for the readout. By using a vertical bichromatic superlattice, we gain full control over coupled layers to implement a charge pump [29-34], which makes our scheme especially robust and efficient. We use this quantum pump to separate two initially coupled layers over large distances. The large separation between the layers, far beyond the depth of focus of our imaging system, enables single-site fluorescence imaging of a single layer without a significant background contribution from the other layer. By using a vertical magnetic field gradient to split different hyperfine states of atoms into different layers in an initial step, we can use the scheme to implement highfidelity spin- and density-resolution of two-dimensional Fermi-Hubbard systems. We first benchmark the pumping and bilayer readout technique by imaging the individual site occupations of a bilayer system, consisting of two coupled two-dimensional Mott insulators. Next, we demonstrate full spin- and density-resolution of a single FermiHubbard layer and reveal strong antiferromagnetic spin correlations in the Mott insulating regime.

Our quantum gas microscope realizes spin- $1 / 2$ FermiHubbard systems using ${ }^{6} \mathrm{Li}$ in a square optical lattice of spacing $a_{x y}=1.15 \mu \mathrm{m}$ and tunnel coupling $t$ in the $x y$ direction [35]. In the vertical $z$ direction the atomic system is usually confined to a single layer of a highly stable bichromatic optical superlattice [see Fig. 1(a) and the Supplemental Material [36] ]. This vertical lattice exhibits short (long) lattice spacings of $a_{z}^{s}=3 \mu \mathrm{m}\left(a_{z}^{l}=6 \mu \mathrm{m}\right)$ and is created by interfering two laser beams of wavelength $\lambda^{s}=532 \mathrm{~nm}\left(\lambda^{l}=1064 \mathrm{~nm}\right)$ at an angle of $5.1^{\circ}$. The phase difference $\phi_{\mathrm{SL}}$ between the two $532 \mathrm{~nm}$ lattice beams is controlled by shifting the frequency of the $532 \mathrm{~nm}$ light, thereby enabling full and dynamical control of the resulting superlattice potential (see the Supplemental Material [36]). Strongly coupled bilayer systems with tunnel couplings of up to $t_{z} / h=876(1) \mathrm{Hz}$ between two layers can be realized with typical lattice depths of $V^{s}=11 E_{R}^{s}$ and $V^{l}=100 E_{R}^{l}$, where $E_{R}^{i}$ denotes the respective recoil energy. A superlattice phase stability of $<25 \mathrm{mrad}$ (see the Supplemental Material [36]) paves the way for coherent bilayer physics in the future.

An important feature of time-modulated superlattices is the existence of two distinct bands $G$ and $E$ with opposite Chern numbers $[29,33,37]$. These bands cause transport in opposite directions upon the same adiabatic passage of the double-well tilt $\Delta$. This is referred to as geometric pumping and lies at the heart of topological charge pumping in

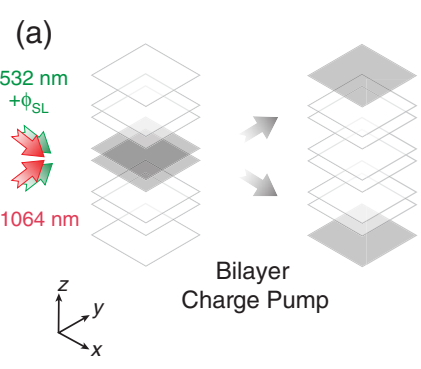

(b)

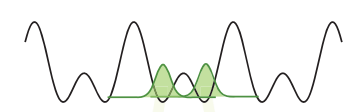

(c)
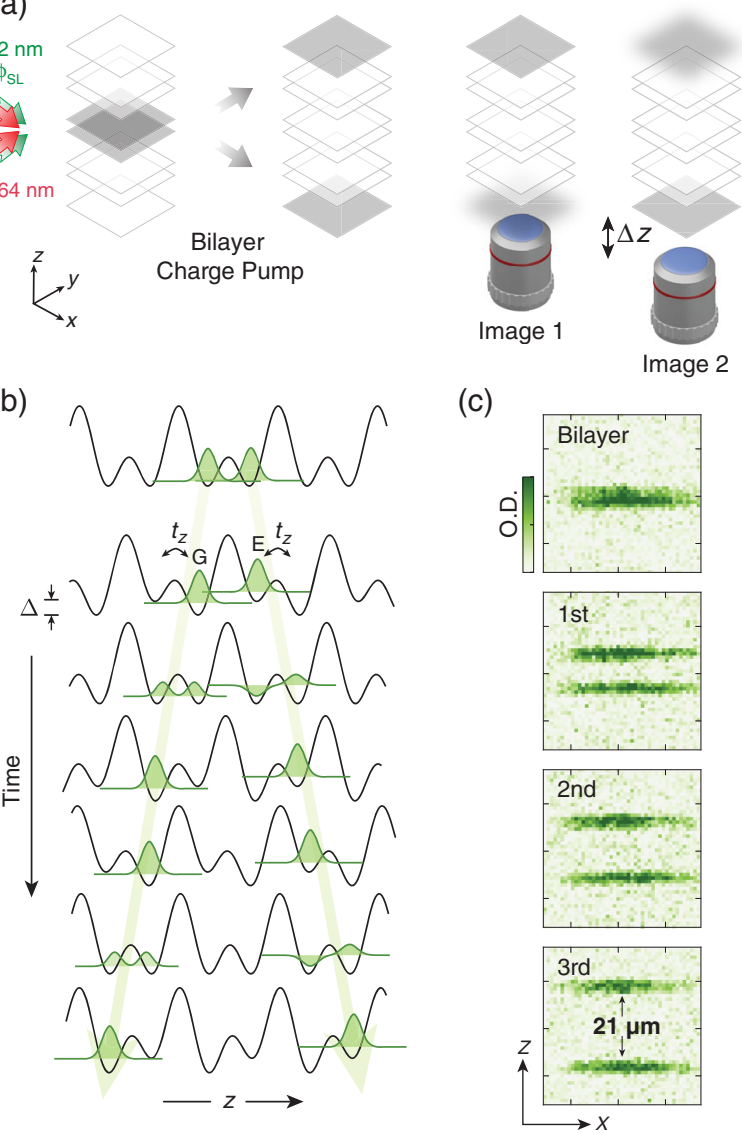

FIG. 1. Schematics of setup and charge pump. (a) We use a bichromatic optical superlattice (red, green) in the vertical direction of our quantum gas microscope to control bilayer systems (gray) and their mutual tunnel couplings $t_{z}$ or energy offsets $\Delta$. The two layers of a bilayer system are pumped to a separation of $21 \mu \mathrm{m}$, which enables subsequent site-resolved microscopy of both layers by shifting the focal plane by $\Delta z$ between the images. (b) Charge pumping is achieved by timedependent modulation of superlattice parameters. Atoms initialized in opposite wells $(G$ and $E$ ) experience transport in opposite directions. For technical reasons we reset the superlattice phase after each adiabatic passage (see the Supplemental Material [36]). (c) Absorption images (side view) of an initially coupled bilayer system whose planes are subsequently pumped in opposite directions for 1,2 , and 3 pumping steps, leading to a final separation of $21 \mu \mathrm{m}$ between the two layers.

time-modulated superlattices [30-34]. As depicted in Fig. 1(b), an atom initialized in $G$ can be transferred to a neighboring well by adiabatically changing the energy offset $\Delta$ between the wells at a constant interwell tunnel coupling $t_{z}$. For the same ramp, an atom in $E$ will be transported to a neighboring well in the opposite direction. Performing $n$ such adiabatic pumping steps can be used to separate two layers, initially displaced by only $a_{z}^{s}$, over macroscopic distances of $a_{z}^{s}+n a_{z}^{l}$. In Fig. 1(c), we show absorption images of atoms initialized in neighboring layers of the vertical superlattice. Initially, the distance 
between the two layers is unresolved, however, after three adiabatic pumping cycles we are able to clearly resolve the wide separation of $21 \mu \mathrm{m}$. Optimized pump parameters for high-fidelity transport are described in the Supplemental Material [36].

A large separation between planes enables the independent microscopy of each layer. Our imaging system $(\mathrm{NA}=0.5)$ exhibits a depth of focus below $3 \mu \mathrm{m}$. When taking a fluorescence image with one layer in focus, the other layer will be out of focus and only contribute a weak and homogeneous background. After capturing the first image, we shift the focal plane of our imaging system to take an image of the other layer. The parasitic background of the opposite layer is extremely weak, such that no further image processing is necessary and occupations can be reconstructed with high fidelity using our usual deconvolution algorithm [22]. To demonstrate the independent readout of two layers, we initialize and image a weakly coupled $\left(t_{z} / t=1.3, \Delta=0\right)$ Mott-insulating bilayer system. After freezing the atomic occupations, three pumping steps are applied to separate the atomic planes. Then we take fluorescence images of both layers with an exposure of $2 \mathrm{~s}$ per picture and reconstruct the single-site resolved occupations (see Fig. 2). Parity projection is avoided through the use of a dedicated pinning lattice [35]. Furthermore, the combined occupation between vertically neighboring sites (forming a supersite) is highly relevant for bilayer systems, as a suppression of density fluctuations on such a supersite is expected for band-insulating [38] or dimer phases [39]. This observable is now readily accessible [see Fig. 2(d)] as well as three-dimensional charge correlators to study bilayer physics in the future.

In order to determine the probability of adiabatic transfer per pumping step $\eta$, we track the number of atoms initialized in a single layer for a variable amount of pumping steps $n \in[0,3,6,9]$. We reverse the pump direction after the third pump to avoid leaving the cooling region of our pinning lattice. Fitting an exponential $\eta^{n}$ to this curve yields a very high pumping efficiency of $\eta=0.996(1)$, underlining the robustness of the separation process. The main limitation of this fidelity is caused by the residual harmonic confinement along the vertical direction of order $\simeq 300 \mathrm{~Hz}$, caused by the $x y$ lattices. It detunes distant double-well structures appreciably compared to the superlattice energy scales $t_{z}$ and reduces the fidelity of pumping in both directions.

The bilayer readout scheme presented above can be readily extended to obtain full spin and density resolution of a single 2D Fermi-Hubbard plane. After realizing such a single two-dimensional system, we first freeze the motion of the atoms in a deep $x y$ lattice and confine them in a single layer of the large-scale vertical lattice. A strong vertical magnetic field gradient of $45 \mathrm{G} / \mathrm{cm}$ is then applied to pull the two spins in opposite directions and the short spaced vertical lattice is turned on adiabatically with a
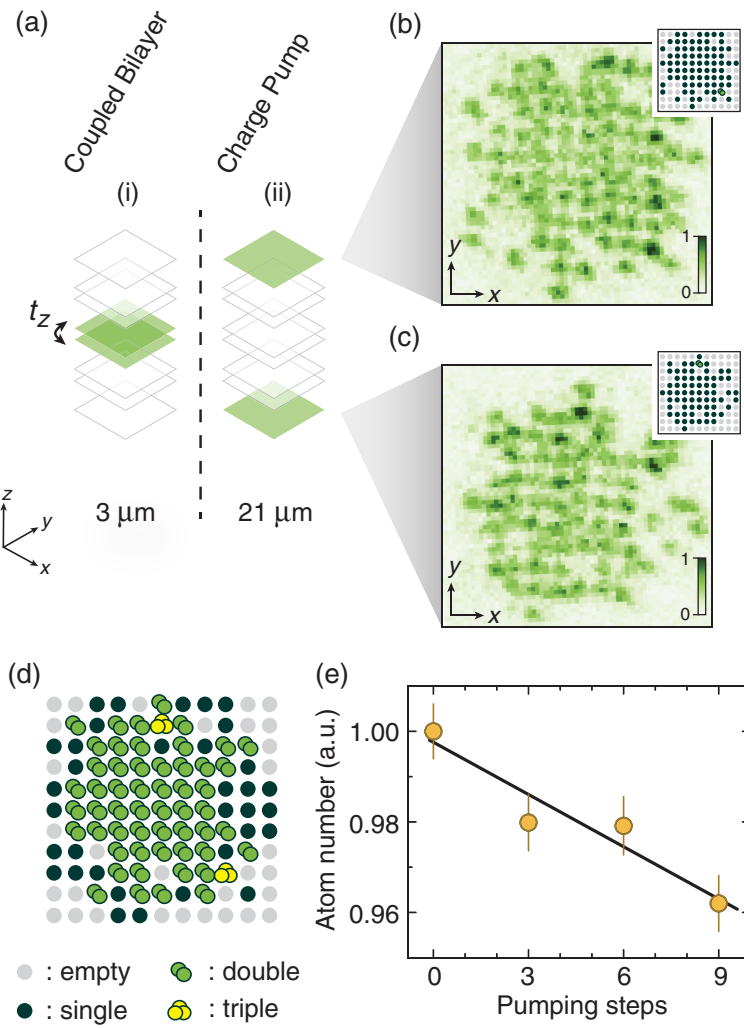

(c)

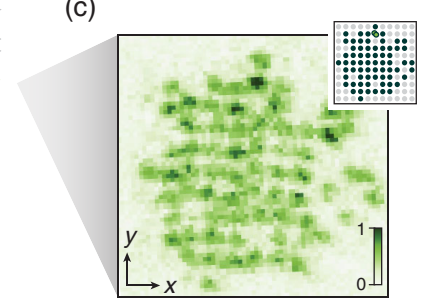

(d)

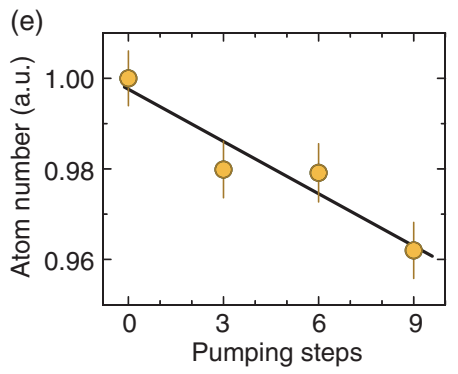

FIG. 2. Bilayer readout and charge pump fidelity. (a) An initially coupled Mott insulating bilayer system is separated over a distance of $21 \mu \mathrm{m}$ using three charge pumping steps. (b), (c), Single-site resolved fluorescence images and reconstruction (inset) of the respective site occupations of the two separated layers. The images were obtained by shifting the high-resolution objective to sequentially image the two layers. (d) Summed occupation of vertically combined sites (supersites) relevant for bilayer systems. Here, the two vertically overlapping Mott insulators show a large region of double occupation on a supersite. (e) Averaged and normalized number of atoms in a monolayer system as a function of pumping steps. A fit to the data (black line) yields a transfer fidelity for each pumping step of $\eta=0.996(1)$. Error bars denote one standard error of the mean (s.e.m.).

phase $\phi_{\mathrm{SL}}$ set to obtain symmetric double wells $(\Delta=0)$ along the vertical direction. We thereby realize a controlled Stern-Gerlach separation [22] of the two spin components into two adjacent planes of the bilayer optical lattice (see Fig. 3). For our experiment, we employ a magnetic offset field below $13 \mathrm{G}$ to maximize the differential magnetic moments between the lowest hyperfine states of ${ }^{6} \mathrm{Li}$ during the splitting process. Once the spin states are split into different layers, we separate the two planes over larger distances using the bilayer readout sequence introduced above. We measure the occupation in each layer as a function of the superlattice phase $\phi_{\mathrm{SL}}$ during the splitting to demonstrate the accuracy of the spin splitting [see Fig. 3(e)]. Working with a spin-balanced system, successful spin splitting manifests itself as a broad range of 


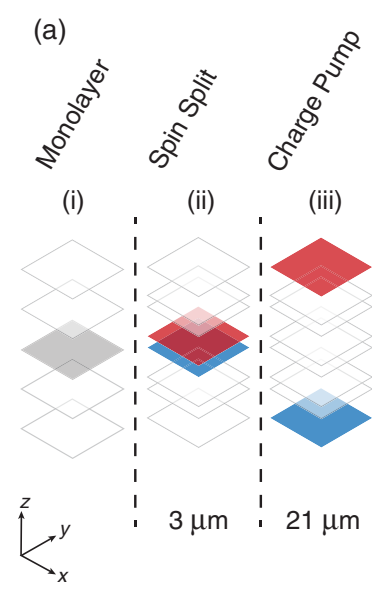

(b)

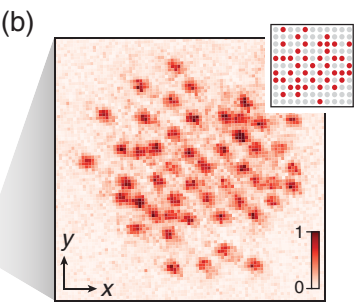

(c)

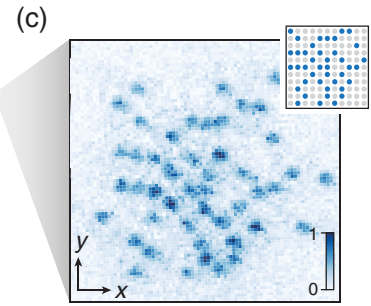

(d)
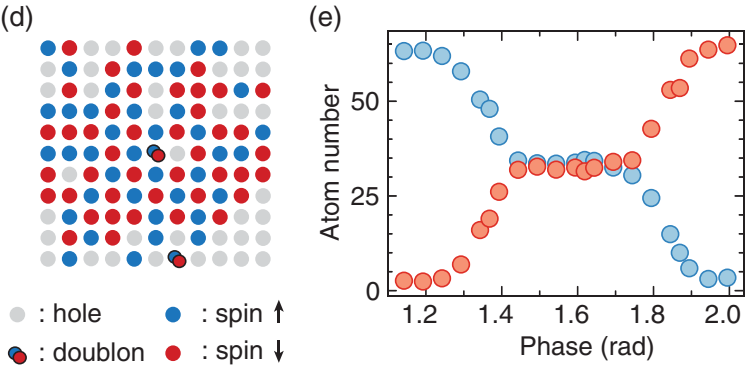

FIG. 3. Spin resolution of two-dimensional systems. (a) The two different spin states of a monolayer spin mixture (here a twodimensional Fermi-Hubbard system) are split into different layers (red and blue) of a bilayer system with a vertical magnetic gradient. Subsequently, charge pumping and bilayer readout is applied with single-site resolution. (b),(c), Snapshot of the two spin components of a two-dimensional Fermi-Hubbard system after separation and the full spin and density reconstruction of the original single layer in (d). The correct recombination of the spinlayers is ensured by the unique minimization of reconstructed doublons (see the Supplemental Material [36]). (e) Number of atoms detected in the upper (red) or lower (blue) layer as a function of the superlattice splitting phase. The magnetic field gradient during the initial splitting process creates a broad plateau of superlattice phases $\phi_{\mathrm{SL}}$, for which a balanced spin mixture is successfully split into its constituents.

superlattice phases $\phi_{\mathrm{SL}}$, for which the occupation in both layers is constant. The width of this plateau is determined by the strength of the magnetic field gradient and the lattice parameters along the $z$ direction. Combining the spinresolved occupations of each layer, we obtain the full density and spin reconstruction of the two-dimensional system [see Fig. 3(d)]. The excellent agreement between densities and density fluctuations of unpumped and spinresolved pumped layers excludes the presence of any significant transverse motion caused by the vertical splitting and pumping (see the Supplemental Material [36]).

The most stringent benchmark, however, for our spinresolution technique is the measurement of spin correlations in a two-dimensional system. For undoped Mott insulators,

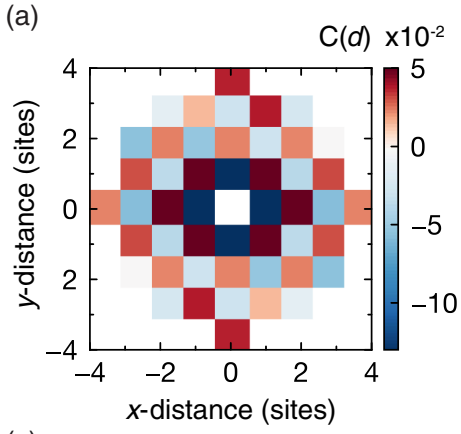

(b)

(c)
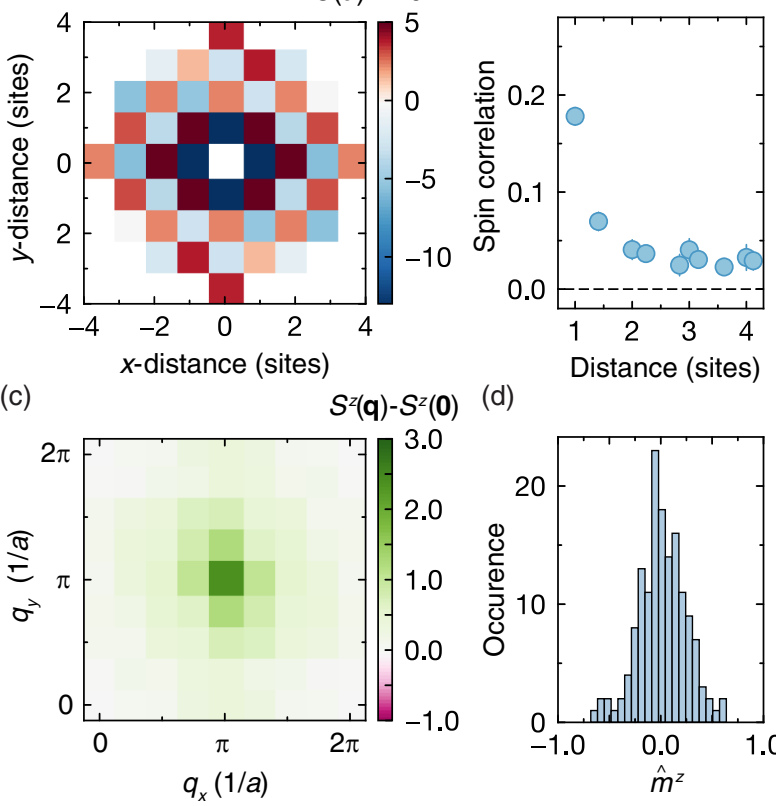

(d)

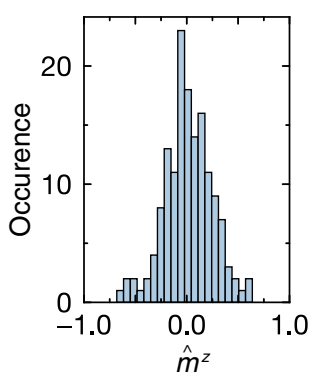

FIG. 4. Two-dimensional spin correlations. (a) Averaged spin correlations versus distance of undoped two-dimensional FermiHubbard systems at $U / t=9.3(4)$ obtained by spin- and densityresolved imaging. Sites with density $n<0.84$ are excluded from the analysis. (b) Sign-corrected spin correlations versus radial distance. Error bars denote one s.e.m. (c) Spin structure-factor, exhibiting a strong peak at the antiferromagnetic momentum $(\pi, \pi)$. (d) Full counting statistics of the staggered magnetization $\hat{m}^{z}$. The figure is based on 150 experimental realizations.

strong antiferromagnetic correlations are expected to arise in the system in the regime of large on site interaction versus tunneling $U / t$ and for low enough temperatures, due to the antiferromagnetic Heisenberg spin couplings $J$. We take spin- and density-resolved measurements of an undoped system at an in-plane lattice depth of 6.9(1) $E_{R}^{x y}$ and $U / t=$ $9.3(4)$ with around 90 atoms. To ensure perfect recovering of the two-dimensional parent system from the spin-layers, we match both layers based on the unique minimization of reconstructed doublons (see the Supplemental Material [36]). At our coldest temperatures, we indeed observe strong antiferromagnetic correlations for our system extending over multiple sites [see Fig. 4(a)]. From the data, we extract spin correlations $C(d)=4\left\langle\hat{S}_{i}^{z} \hat{S}_{i+d}^{z}\right\rangle$ as well as reveal a strong peak in the corresponding spin structure factor $S(\mathbf{q})$ at the antiferromagnetic quasimomentum $\mathbf{q}=(\pi, \pi)$. We find a mean staggered magnetization of $m^{z}=\sqrt{\left\langle\left(\hat{m}^{z}\right)^{2}\right\rangle}=$ $0.19(1)$ in our system (see the Supplemental Material [36]). Comparing with Quantum Monte Carlo calculations of [10] at $U / t=7.2$, these correlations correspond to a temperature of $k_{B} T \simeq 0.6 \mathrm{~J}$, where $k_{B}$ is the Boltzmann constant. Such temperatures are among the lowest that have been reported for ultracold fermionic systems and underline 
that the detection process employed preserves the intricate correlations in the system.

Our work underlines the unique detection possibilities afforded by versatile and highly controlled superlattice setups for quantum gas microscopy. Next to realizing tunable bilayer systems with independent density readout, we showed how our scheme can be used as a universal technique to obtain full spin and density resolution of single 2D Fermi-Hubbard layers for arbitrary 2D lattice geometries. The methods demonstrated here combine robust charge pumping and efficient Stern-Gerlach separation to provide an entirely new degree of control and readout for quantum gas microscopes. Our work extends the capabilities of such systems to coupled layered systems, which are highly relevant in the context of high- $T_{c}$ superconductivity $[38,40-43]$. An extension of the scheme to spin detection of a bilayer system requires four images and can be implemented in the future. Bilayer charge pumping can be applied to continuous quantum gases or combined with other imaging techniques. Furthermore, advanced cooling schemes for two-dimensional systems based on dynamical disentangling of layers are now within reach [44], and schemes for nontrivial observables like entanglement entropy $[45,46]$ or angle-resolved-photoemission spectroscopy $[47,48]$ can be realized on the repulsive side $(U>0)$, and for higher dimensional systems, based on the technique reported here.

This work was supported by the Max Planck Society (MPG), the European Union (Grant No. FET-Flag 817482, PASQUANS), the Max Planck Harvard Research Center for Quantum Optics (MPHQ) and under Germany's Excellence Strategy-EXC-2111—Grant No. 39081486. J. K. gratefully acknowledges funding from Hector Fellow Academy. We would like to thank Michael Höse for early contributions to the superlattice setup.

*joannis.koepsell@mpq.mpg.de

[1] C. Gross and I. Bloch, Science 357, 995 (2017).

[2] I. M. Georgescu, S. Ashhab, and F. Nori, Rev. Mod. Phys. 86, 153 (2014).

[3] R. Blatt and C. F. Roos, Nat. Phys. 8, 277 (2012).

[4] H. Weimer, M. Müller, I. Lesanovsky, P. Zoller, and H. P. Büchler, Nat. Phys. 6, 382 (2010).

[5] M. H. Devoret and R. J. Schoelkopf, Science 339, 1169 (2013).

[6] P. A. Lee, N. Nagaosa, and X.-G. Wen, Rev. Mod. Phys. 78, 17 (2006).

[7] B. Keimer, S. A. Kivelson, M. R. Norman, S. Uchida, and J. Zaanen, Nature (London) 518, 179 (2015).

[8] A. Damascelli, Z. Hussain, and Z. X. Shen, Rev. Mod. Phys. 75, 473 (2003).

[9] O. Yuli, I. Asulin, O. Millo, D. Orgad, L. Iomin, and G. Koren, Phys. Rev. Lett. 101, 057005 (2008).
[10] A. Mazurenko, C. S. Chiu, G. Ji, m. F. Parsons, M. KanászNagy, R. Schmidt, F. Grusdt, E. Demler, D. Greif, and M. Greiner, Nature (London) 545, 462 (2017).

[11] M. A. Nichols, L. W. Cheuk, M. Okan, T. R. Hartke, E. Mendez, T. Senthil, E. Khatami, H. Zhang, and M. W. Zwierlein, Science 363, 383 (2019).

[12] P. T. Brown, D. Mitra, E. Guardado-Sanchez, R. Nourafkan, A. Reymbaut, C. D. Hébert, S. Bergeron, A. M. Tremblay, J. Kokalj, D. A. Huse, P. Schauß, and W. S. Bakr, Science 363, 379 (2019).

[13] C. S. Chiu, G. Ji, A. Bohrdt, M. Xu, M. Knap, E. Demler, F. Grusdt, M. Greiner, and D. Greif, Science 365, 251 (2019).

[14] W. S. Bakr, J. I. Gillen, A. Peng, S. Fölling, and M. Greiner, Nature (London) 462, 74 (2009).

[15] J. F. Sherson, C. Weitenberg, M. Endres, M. Cheneau, I. Bloch, and S. Kuhr, Nature (London) 467, 68 (2010).

[16] M. F. Parsons, A. Mazurenko, C. S. Chiu, G. Ji, D. Greif, and M. Greiner, Science 353, 1253 (2016).

[17] L. W. Cheuk, M. A. Nichols, K. R. Lawrence, M. Okan, H. Zhang, E. Khatami, N. Trivedi, T. Paiva, M. Rigol, and M. W. Zwierlein, Science 353, 1260 (2016).

[18] T. A. Hilker, G. Salomon, F. Grusdt, A. Omran, M. Boll, E. Demler, I. Bloch, and C. Gross, Science 357, 484 (2017).

[19] G. Salomon, J. Koepsell, J. Vijayan, T. A. Hilker, J. Nespolo, L. Pollet, I. Bloch, and C. Gross, Nature (London) 565, 56 (2019).

[20] J. Vijayan, P. Sompet, G. Salomon, J. Koepsell, S. Hirthe, A. Bohrdt, F. Grusdt, I. Bloch, and C. Gross, Science 367, 186 (2020).

[21] J. Koepsell, J. Vijayan, P. Sompet, F. Grusdt, T. A. Hilker, E. Demler, G. Salomon, I. Bloch, and C. Gross, Nature (London) 572, 358 (2019).

[22] M. Boll, T. A. Hilker, G. Salomon, A. Omran, J. Nespolo, L. Pollet, I. Bloch, and C. Gross, Science 353, 1257 (2016).

[23] T. Y. Wu, A. Kumar, F. Giraldo, and D. S. Weiss, Nat. Phys. 15, 538 (2019).

[24] M. Kwon, M. F. Ebert, T. G. Walker, and M. Saffman, Phys. Rev. Lett. 119, 180504 (2017).

[25] K. D. Nelson, X. Li, and D. S. Weiss, Nat. Phys. 3, 556 (2007).

[26] D. Barredo, V. Lienhard, S. de Léséleuc, T. Lahaye, and A. Browaeys, Nature (London) 561, 79 (2018).

[27] O. Elíasson, R. Heck, J. S. Laustsen, R. Müller, C. A. Weidner, J. J. Arlt, and J. F. Sherson, arXiv:1912.03079.

[28] P. M. Preiss, R. Ma, M. E. Tai, J. Simon, and M. Greiner, Phys. Rev. A 91, 041602(R) (2015).

[29] D. J. Thouless, Phys. Rev. B 27, 6083 (1983).

[30] O. Romero-Isart and J. J. García-Ripoll, Phys. Rev. A 76, 052304 (2007).

[31] Y. Qian, M. Gong, and C. Zhang, Phys. Rev. A 84, 013608 (2011).

[32] L. Wang, M. Troyer, and X. Dai, Phys. Rev. Lett. 111, 026802 (2013).

[33] M. Lohse, C. Schweizer, O. Zilberberg, M. Aidelsburger, and I. Bloch, Nat. Phys. 12, 350 (2016).

[34] S. Nakajima, T. Tomita, S. Taie, T. Ichinose, H. Ozawa, L. Wang, M. Troyer, and Y. Takahashi, Nat. Phys. 12, 296 (2016).

[35] A. Omran, M. Boll, T. A. Hilker, K. Kleinlein, G. Salomon, I. Bloch, and C. Gross, Phys. Rev. Lett. 115, 263001 (2015). 
[36] See the Supplemental Material at http://link.aps.org/ supplemental/10.1103/PhysRevLett.125.010403 for details on the superlattice, the experimental sequence and data analysis.

[37] M. Atala, M. Aidelsburger, J. T. Barreiro, D. Abanin, T. Kitagawa, E. Demler, and I. Bloch, Nat. Phys. 9, 795 (2013).

[38] M. Golor, T. Reckling, L. Classen, M. M. Scherer, and S. Wessel, Phys. Rev. B 90, 195131 (2014).

[39] R. Chen, S. B. Lee, and L. Balents, Phys. Rev. B 87, 161119 (R) (2013).

[40] T. A. Maier and D. J. Scalapino, Phys. Rev. B 84, 180513(R) (2011).

[41] A. I. Liechtenstein, I. I. Mazin, and O. K. Andersen, Phys. Rev. Lett. 74, 2303 (1995).
[42] N. Bulut, D. J. Scalapino, and R. T. Scalettar, Phys. Rev. B 45, 5577 (1992).

[43] S. Okamoto and T. A. Maier, Phys. Rev. Lett. 101, 156401 (2008).

[44] A. Kantian, S. Langer, and A. J. Daley, Phys. Rev. Lett. 120, 060401 (2018).

[45] H. Pichler, L. Bonnes, A. J. Daley, A. M. Läuchli, and P. Zoller, New J. Phys. 15, 063003 (2013).

[46] R. Islam, R. Ma, P. M. Preiss, M. E. Tai, A. Lukin, M. Rispoli, and M. Greiner, Nature (London) 528, 77 (2015).

[47] A. Bohrdt, D. Greif, E. Demler, M. Knap, and F. Grusdt, Phys. Rev. B 97, 125117 (2018).

[48] P. T. Brown, E. Guardado-Sanchez, B. M. Spar, E. W. Huang, T. P. Devereaux, and W. S. Bakr, Nat. Phys. 16, $26(2020)$. 